\title{
Spherical Scanning 3-D Microwave Imaging of Near-field Scatterers Mutually Coupled With an Antenna Array
}

This paper was downloaded from TechRxiv (https://www.techrxiv.org).

LICENSE

CC BY 4.0

SUBMISSION DATE / POSTED DATE

$30-10-2021 / 02-11-2021$

\section{CITATION}

Watanabe, Takuma; Yamada, Hiroyoshi (2021): Spherical Scanning 3-D Microwave Imaging of Near-field Scatterers Mutually Coupled With an Antenna Array. TechRxiv. Preprint.

https://doi.org/10.36227/techrxiv.16893517.v1

$\mathrm{DOI}$

10.36227/techrxiv.16893517.v1 


\title{
Spherical Scanning 3-D Microwave Imaging of Near-field Scatterers Mutually Coupled With an Antenna Array
}

\author{
Takuma Watanabe and Hiroyoshi Yamada, Member, IEEE
}

\begin{abstract}
In this article, we propose a 3-D synthetic aperture imaging method with spherical antenna scanning to identify scatterers located close to an antenna array, such as fixtures or support of the antenna. Previous studies have shown that 2$D$ and 3-D synthetic aperture imaging techniques with planar, circular, and cylindrical scanning can successfully reconstruct spatial images of near-field scatterers. The spherical scanning approach considered in this article is expected to improve the 3-D image resolution because more angular diversity can be achieved in the elevation direction. However, as we show in this study, simple extension of the previous techniques to the spherical case results in undesired blur artifacts in the reconstructed image. To overcome this problem, we introduce a correction factor in the image reconstruction. The proposed imaging algorithm is validated by numerical electromagnetic simulation based on the method of moments.
\end{abstract}

Index Terms-Antenna arrays, mutual coupling, synthetic aperture imaging, bistatic radar, spherical scanning, 3-D image.

\section{INTRODUCTION}

$\mathbf{M}$ ICROWAVE imaging has been widely studied and utilized for various applications ranging from near-field to far-field regions, such as airborne radar imaging [1], [2], nondestructive testing [4]-[6], subsurface imaging [3], radar cross-section measurement [7]-[9], and antenna measurement [10]-[13], which is discussed in this study.

For antenna measurement applications, microwave imaging techniques have been proposed to determine the locations around the antenna from which strong scattering is produced [10]-[13]. For example, antenna fixtures, support structures, and radomes are possible sources of strong scattering. This scattering often causes undesired effects, such as altering the antenna pattern and degrading the performance of array signal processing, such as direction-of-arrival estimation; these secondary radiations are also known as mutual coupling. Therefore, determination of the location and strength of the scattering is required in the antenna design and testing process.

In previous studies, 2-D and 3-D microwave imaging techniques with planar [11], circular [12], and cylindrical apertures [13] were demonstrated for the aforementioned purpose. These

Manuscript received $\mathrm{xxxx} \mathrm{xx}, \mathrm{xxxx}$; revised $\mathrm{xxxx} \mathrm{xx}, \mathrm{xxxx}$; accepted $\mathrm{xxxx}$ $\mathrm{xx}, \mathrm{xxxx}$. Date of publication $\mathrm{xxxx} \mathrm{xx}, \mathrm{xxxx}$; date of current version $\mathrm{xxxx}$ $\mathrm{xx}, \mathrm{xxxx}$, The Associate Editor coordinating the review process was X. Xxxx. (Corresponding author: Takuma Watanabe.)

T. Watanabe is currently with Fujitsu Ltd., Kawasaki 2118588, Japan (email: takuma.watanabe.jp@ieee.org).

H. Yamada is with the Smart Information Systems Program, Faculty of Engineering, Niigata University, Niigata 9502181, Japan.

Digital Object Identifier xxxxx/xxxxx methods are based on bistatic synthetic aperture imaging, where a probe antenna is moved around another antenna under test (AUT) with near-field scatterers that are assumed to be irremovable from the structure of the AUT. Either the probe antenna or the AUT transmits an electromagnetic wave, and the other antenna collects scattered waves from the near-field scatterers. The measurement is performed at various locations within the synthetic aperture, and then, the measured data are synthesized to reconstruct a 2-D or 3-D spatial image.

Based on these previous works, we consider an extension of the conventional methods to a spherical scanning case in this article. This approach is expected to improve the 3D image resolution because more angular diversity can be achieved in the elevation direction. However, as discussed for the monostatic case in [9], a simple extension of the previous techniques to a spherical case results in undesired blur artifacts in the reconstructed image. To overcome this problem, a correction factor was introduced for the image reconstruction in [9]. Our objective is to show the validity of this correction factor for bistatic synthetic aperture imaging to determine the sources of undesired scattering.

The main novelty and contribution of this study are to show the extension of the previous circular and cylindrical synthetic aperture imaging techniques to a spherical scanning case. We show that a simple extension of the previous approaches to a spherical case is infeasible because of significant blur artifacts in the reconstructed image. To reduce undesired artifacts, we apply a correction factor in the image reconstruction process, which was originally developed for monostatic radar crosssection determination from synthetic aperture imaging in [9]. Numerical electromagnetic simulation based on the method of moments shows that the correction factor can be applied to the bistatic imaging geometry to reduce the undesired blur artifacts in the reconstructed image.

\section{PRoblem Formulation}

\section{A. System and Signal Model}

Fig. 1 depicts the system model considered in this article. An AUT is an antenna array with near-field scatterers, and the array elements and the near-field scatterers are distributed over the 3-D spatial domain $(x, y, z)$. We denote the element location as $\boldsymbol{r}_{m}, m \in\{1,2, \ldots\}$ and the scatter location as $\boldsymbol{s}_{n}, n \in\{1,2, \ldots\}$, where $n$ and $m$ are indices to represent the individual array element and the scatterer. Note that the near-field scatterers are assumed to be irremovable from the AUT because these are parts of the antenna structure. 


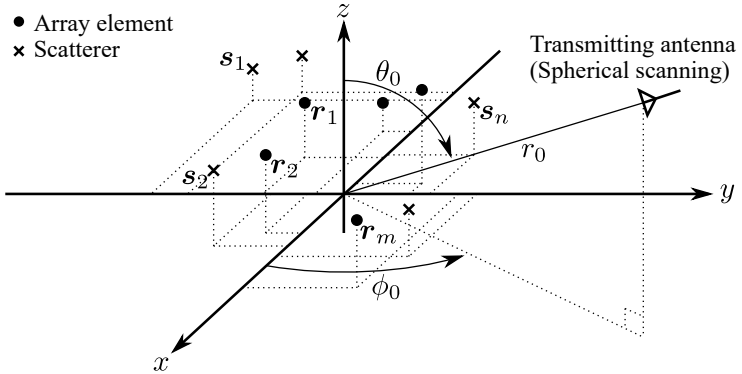

Fig. 1. System model of spherical scanning measurement of an antenna array with near-field scatterers.

These array elements and the scatterers are irradiated by the transmitting antenna located at $\boldsymbol{r}_{0}=\left(x_{0}, y_{0}, z_{0}\right)$. The distance between the origin and the transmitting antenna is fixed and denoted by $r_{0}$. The transmitting antenna is scanned in both azimuth and zenith directions, denoted by $\phi_{0}$ and $\theta_{0}$, respectively. Using these notations, the location of the transmitting antenna can be expressed as

$$
\begin{aligned}
& x_{0}\left(\phi_{0}, \theta_{0}\right)=r_{0} \cos \phi_{0} \sin \theta_{0} \\
& y_{0}\left(\phi_{0}, \theta_{0}\right)=r_{0} \sin \phi_{0} \sin \theta_{0} \\
& z_{0}\left(\phi_{0}, \theta_{0}\right)=r_{0} \cos \theta_{0} .
\end{aligned}
$$

For later discussion, we also define the vector $\boldsymbol{R}$ from an arbitrary spatial location $\boldsymbol{r}=(x, y, z)$ to the transmitting antenna location $\boldsymbol{r}_{0}$ as follows:

$$
\begin{aligned}
\boldsymbol{R}\left(\phi_{0}, \theta_{0}\right) & =\boldsymbol{r}_{0}-\boldsymbol{r}=R_{x} \widehat{\boldsymbol{x}}+R_{y} \widehat{\boldsymbol{y}}+R_{z} \widehat{\boldsymbol{z}} \\
R_{x}\left(\phi_{0}, \theta_{0}\right) & =x_{0}\left(\phi_{0}, \theta_{0}\right)-x \\
R_{y}\left(\phi_{0}, \theta_{0}\right) & =y_{0}\left(\phi_{0}, \theta_{0}\right)-y \\
R_{z}\left(\phi_{0}, \theta_{0}\right) & =z_{0}\left(\phi_{0}, \theta_{0}\right)-z,
\end{aligned}
$$

where $\left(R_{x}, R_{y}, R_{z}\right)$ can be considered as the transmitting antenna location represented in a local coordinate system centered at the spatial location $\boldsymbol{r}$. Moreover, we define the local azimuth and zenith angles of the transmitting antenna in the local coordinate system, denoted by $\alpha$ and $\beta$, respectively, as

$$
\begin{aligned}
\alpha\left(\phi_{0}, \theta_{0}\right) & =\tan ^{-1}\left[R_{y}\left(\phi_{0}, \theta_{0}\right) / R_{x}\left(\phi_{0}, \theta_{0}\right)\right] \\
\beta\left(\phi_{0}, \theta_{0}\right) & =\tan ^{-1}\left[\rho / R_{z}\left(\phi_{0}, \theta_{0}\right)\right] \\
\rho\left(\phi_{0}, \theta_{0}\right) & =\sqrt{R_{x}^{2}\left(\phi_{0}, \theta_{0}\right)+R_{y}^{2}\left(\phi_{0}, \theta_{0}\right)} .
\end{aligned}
$$

Under the aforementioned measurement configuration, the transmitting antenna repeatedly transmits an electromagnetic wave at each spherical scanning location $\left(\phi_{0}, \theta_{0}\right)$, and the receiving array elements collect the scattered wave from the near-field scatterers.

We formulate the received signal of the $m$ th array element at wavenumber $k$ and transmitting antenna location $\boldsymbol{r}_{0}$ as the sum of direct and scattered wave components. For simplicity, we assume that all of the array elements have an isotropic antenna pattern. Therefore, the received signal for the $m$ th array element is expressed as

$$
\begin{aligned}
E_{m}\left(k, \boldsymbol{r}_{0}\right)= & E_{m}^{d}\left(k, \boldsymbol{r}_{0}\right)+E_{m}^{s}\left(k, \boldsymbol{r}_{0}\right), \\
E_{m}^{d}\left(k, \boldsymbol{r}_{0}\right)= & \frac{A\left(k, \boldsymbol{r}_{0}, \boldsymbol{r}_{m}\right)}{\left|\boldsymbol{r}_{0}-\boldsymbol{r}_{m}\right|} e^{-j k\left|\boldsymbol{r}_{0}-\boldsymbol{r}_{m}\right|}, \\
E_{m}^{s}\left(k, \boldsymbol{r}_{0}\right)= & \int_{\Omega} \frac{A\left(k, \boldsymbol{r}_{0}, \boldsymbol{r}\right)}{|\boldsymbol{R}|} e^{-j k|\boldsymbol{R}|} \\
& \cdot \frac{f\left(k, \boldsymbol{r}_{0}, \boldsymbol{r}, \boldsymbol{r}_{m}\right)}{\left|\boldsymbol{r}-\boldsymbol{r}_{m}\right|} e^{-j k\left|\boldsymbol{r}-\boldsymbol{r}_{m}\right|} d \boldsymbol{r},
\end{aligned}
$$

where $E_{m}^{d}\left(k, \boldsymbol{r}_{0}\right)$ and $E_{m}^{s}\left(k, \boldsymbol{r}_{0}\right)$ represent the direct and scattered wave components, respectively, and $k=\omega / c$ with the angular frequency $\omega$ and the speed of light $c$. The domain of the integration $\Omega$ is the spatial domain within the scanning sphere. In Eqs. (4b) and (4c), $A\left(k, \boldsymbol{r}_{0}, \cdot\right)$ and $f\left(k, \boldsymbol{r}_{0}, \boldsymbol{r}, \boldsymbol{r}_{m}\right)$ are the complex amplitude of the transmitting and the bistatic scattering coefficient, respectively.

As discussed in [3], [12], [13], the direct wave component $E_{m}^{d}\left(k, \boldsymbol{r}_{0}\right)$ must be suppressed from the received signal $E_{m}\left(k, \boldsymbol{r}_{0}\right)$ before the image reconstruction process. Without removing the direct wave, the weaker scattered wave component is masked by the direct wave component and is unable to locate the near-field scatterers of interest. The detailed process of direct wave filtering is given in [3], [12] and is not repeated here. In the following, we assume that the direct wave component is properly filtered out of the received signal, and we denote the filtered received signal as $E_{m}^{\prime}\left(k, \boldsymbol{r}_{0}\right) \simeq E_{m}^{s}\left(k, \boldsymbol{r}_{0}\right)$.

\section{B. Image Reconstruction}

The image reconstruction can be achieved via the following integral transformation of the filtered received signal $E_{m}^{\prime}\left(k, \boldsymbol{r}_{0}\right)$ of the $m$ th receiving element with a weighting function $F_{m}\left(k, \boldsymbol{r}_{0}\right)$ :

$$
\psi_{m}(\boldsymbol{r})=\int_{0}^{\infty} \int_{0}^{2 \pi} \int_{0}^{\pi} E_{m}^{\prime}\left(k, \boldsymbol{r}_{0}\right) F_{m}\left(k, \boldsymbol{r}_{0}\right) d \theta_{0} d \phi_{0} d k
$$

The weighting function $F_{m}\left(k, \boldsymbol{r}_{0}\right)$ is called the focusing factor that compensates the propagation phase and attenuation and is defined as follows:

$$
F_{m}\left(k, \boldsymbol{r}_{0}, \boldsymbol{r}\right)=g\left(k, \boldsymbol{r}_{0}, \boldsymbol{r}\right)|\boldsymbol{R}|\left|\boldsymbol{r}-\boldsymbol{r}_{m}\right| e^{j k|\boldsymbol{R}|} e^{j k\left|\boldsymbol{r}-\boldsymbol{r}_{m}\right|}
$$

where $g\left(k, \boldsymbol{r}_{0}, \boldsymbol{r}\right)$ is a correction factor and where the conventional method with circular and cylindrical scanning discussed in [12] and [13], respectively, corresponds to $g\left(k, \boldsymbol{r}_{0}, \boldsymbol{r}\right)=1$ (without a correction factor). However, for the case of spherical scanning, the reconstructed image shows undesired artifacts without the correction factor, as shown in [9] and Section III. We can compensate for these artifacts by introducing the following correction factor for a spherical scanning surface [9]:

$$
g\left(k, \boldsymbol{r}_{0}, \boldsymbol{r}\right)=|\sin \beta|\left|\alpha_{\phi_{0}} \beta_{\theta_{0}}-\alpha_{\theta_{0}} \beta_{\phi_{0}}\right|
$$


with

$$
\begin{aligned}
& \alpha_{\phi_{0}}=\frac{\partial \alpha}{\partial \phi_{0}}=\frac{r_{0} \sin \theta_{0}}{\rho^{2}}\left(R_{x} \cos \phi_{0}+R_{y} \sin \phi_{0}\right) \\
& \alpha_{\theta_{0}}= \frac{\partial \alpha}{\partial \theta_{0}}=\frac{r_{0} \cos \theta_{0}}{\rho^{2}}\left(R_{x} \sin \phi_{0}-R_{y} \cos \phi_{0}\right) \\
& \beta_{\phi_{0}}=\frac{\partial \beta}{\partial \phi_{0}}=\frac{R_{z} r_{0} \sin \theta_{0}}{\rho R^{2}}\left(R_{y} \cos \phi_{0}-R_{x} \sin \phi_{0}\right) \\
& \beta_{\theta_{0}}=\frac{\partial \beta}{\partial \theta_{0}}=\frac{r_{0}}{\rho R^{2}}\left[\rho^{2} \sin \theta_{0}+R_{z} \cos \theta_{0}\right. \\
&\left.\cdot\left(R_{x} \cos \phi_{0}+R_{y} \sin \phi_{0}\right)\right] .
\end{aligned}
$$

The correction factor was originally derived in [8] for 2D image reconstruction with circular scanning to improve radar-cross section prediction from near-field measurement and extended to a general 3-D case with an arbitrary scanning surface including a sphere in [9], and the effectiveness of the correction factor of Eq. (7) was proven for a monostatic case. Our purpose is to show that this correction factor can also be applied to the bistatic case to improve the reconstructed image. Because the correction factor derived in [9] can be used for an arbitrary scanning surface, one can readily extend the algorithm presented in this article to another antenna scanning geometry, although we only consider the spherical case in our discussion.

We repeat the aforementioned image reconstruction process for all the array elements. Then, the final image can be created by coherently summing the individual image $\psi_{m}(\boldsymbol{r})$ as

$$
\psi(\boldsymbol{r})=\sum_{m} \psi_{m}(\boldsymbol{r})
$$

\section{NUMERICAL SimULATION}

\section{A. Overview of the Simulation}

We demonstrate the proposed 3-D imaging algorithm using numerical electromagnetic simulation based on the method of moments. Fig. 2 shows the geometry of the simulation, and Table I lists the simulation parameters. A 4-element uniform linear array is placed along the $x$-axis as an AUT, and the array aperture is at the origin of the coordinate system. The array elements are dipoles of which the length and diameter are the half-wavelength $(\lambda / 2$, where $\lambda$ is the wavelength) at the center frequency $(1 \mathrm{GHz})$ and $2 \mathrm{~mm}$, respectively. The element spacing is the half-wavelength. At the center of each array element, a $50-\Omega$ dummy load is connected. The AUT is enclosed by 4 vertical wires having the same length and diameter as the array elements, as shown in Fig. 2. The center of the wire is at $z=0$ for the wires at $s_{1}$ and $s_{4}$ and $z=0.2 \mathrm{~m}$ for the wires at $s_{2}$ and $s_{3}$.

The transmitting antenna is an infinitesimal dipole polarized in the zenith $(\theta)$ direction. The center frequency is $1 \mathrm{GHz}$ and the bandwidth is $40 \mathrm{MHz}$. The distance between the center of the coordinate system and the transmitting antenna is $1.5 \mathrm{~m}$. The angular sampling intervals in the azimuth and zenith directions are both $4^{\circ}$. The angular intervals in the azimuth and zenith directions are $\left[0^{\circ}, 360^{\circ}\right]$ and $\left[0^{\circ}, 180^{\circ}\right]$, respectively. Our purpose is to reconstruct the locations and scattering intensities of these near-field scatterers from the received signal using spherical antenna scanning.

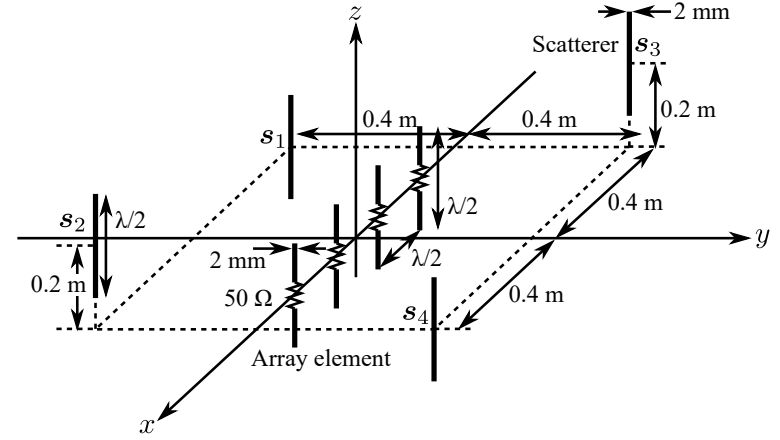

Fig. 2. Geometry of the simulation. A 4-element uniform linear array is enclosed by 4 wire-like scatterers. An infinitesimal dipole is used as the transmitting antenna, and the radius of the scanning sphere is $r_{0}=1.5 \mathrm{~m}$.

TABLE I

SIMULATION PARAMETERS.

\begin{tabular}{l|l}
\hline Receiving array & Uniform linear array \\
Number of array elements & 4 \\
Element & Dipole antenna \\
Element spacing & $\lambda / 2 @ 1 \mathrm{GHz}$ \\
Element diameter & $2 \mathrm{~mm}$ \\
Element length & $\lambda / 2 @ 1 \mathrm{GHz}$ \\
Dummy load & $50 \Omega$ \\
\hline Number of scatterers & 4 \\
Scatterer & Wire \\
Scatterer diameter & $2 \mathrm{~mm}$ \\
Scatterer length & $\lambda / 2 @ 1 \mathrm{GHz}$ \\
\hline Transmitting antenna & Infinitesimal dipole \\
Transmitting antenna distance & $1.5 \mathrm{~m}$ \\
Polarization & $\theta-$ polarization \\
Center frequency & $1 \mathrm{GHz}$ \\
Bandwidth & $400 \mathrm{MHz}$ \\
Frequency sampling interval & $40 \mathrm{MHz}$ \\
Angular sampling interval & $4^{\circ}$ \\
Angular interval in azimuth & {$\left[0^{\circ}, 360^{\circ}\right]$} \\
Angular interval in zenith & {$\left[0^{\circ}, 180^{\circ}\right]$} \\
\hline
\end{tabular}

\section{B. Results and Discussion}

Figs. 3 and 4 show the reconstructed 3-D spatial images without and with the correction factor defined in Eq. (7), respectively. In these figures, (a) shows the $(x, y)$-plane image at $z=0$, and (b) shows the $(x, z)$-plane image at $y=0.4 \mathrm{~m}$. As shown in Fig. 3, strong peaks exist at the array element and the scatter locations. However, the responses at the scatterer locations seem to be blurred, and it is difficult to identify the true location of the scatterer from Fig. 3. Moreover, Fig. 3(a) contains responses from the scatterers of nonzero altitude (i.e., $s_{2}=(0.4,-0.4,0.2) \mathrm{m}$ and $\left.s_{3}=(-0.4,0.4,0.2) \mathrm{m}\right)$, although Fig. 3(a) is a $(x, y)$-plane image at $z=0$. As shown in Fig. 3(b), this is because the responses of the scatterers are blurred in the $z$-direction such that leakage of the response is observed in the $(x, y)$-plane image at $z=0$.

The abovementioned artifact can be reduced by introducing the correction factor. A comparison of Figs. 3 and 4 reveals that the spatial image is improved by the correction factor such that one can readily determine the location and scattering strength of the near-field scatterers. In Fig. 4, the responses of the scatterers are more focused, and unlike the conventional method, the leakage from the scatterers at nonzero altitude is 


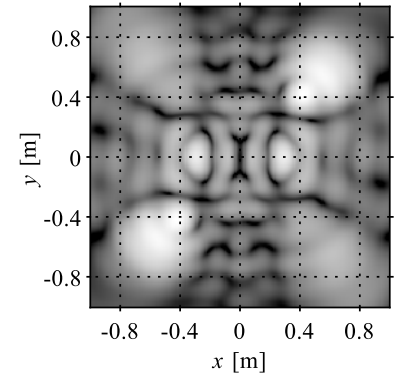

(a)

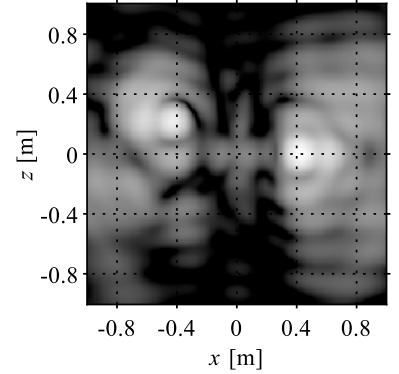

(b)
Fig. 3. Reconstructed 3-D image without the correction factor: (a) $(x, y)$ plane image at $z=0$. (b) $(x, z)$-plane image at $y=0.4 \mathrm{~m}$.

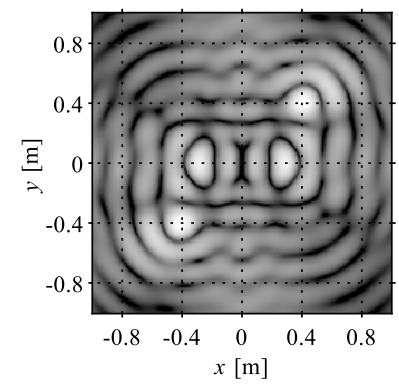

(a)

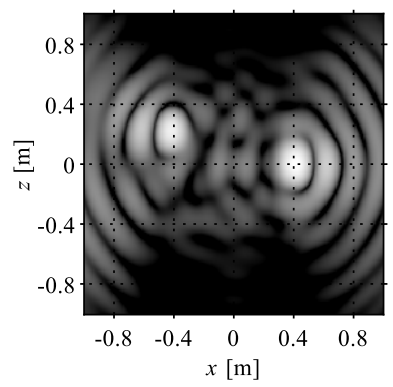

(b)
Fig. 4. Reconstructed 3-D image with the correction factor: (a) $(x, y)$-plane image at $z=0$. (b) $(x, z)$-plane image at $y=0.4 \mathrm{~m}$.

invisible in the $(x, y)$-plane image at $z=0$, as shown in 4(a).

From these simulation results, the proposed spherical scanning 3-D microwave imaging with the correction factor can be used to determine the near-field scatterers located close to an antenna array.

\section{CONCLUSION}

In this article, we propose spherical scanning 3-D microwave imaging to locate the near-field scatterers of an antenna array. We apply a correction factor that was originally introduced for the monostatic synthetic aperture imaging problem and show that this correction factor is also effective for bistatic imaging applications such as those considered in this study. The numerical electromagnetic simulation shows the validity of the proposed algorithm. The proposed method can be used for the design process of antenna arrays with unavoidable near-field scatterers.

\section{REFERENCES}

[1] O. Ponce, P. Prats-Iraola, M. Pinheiro, M. Rodriguez-Cassola, R. Scheiber, A. Reigber, and A. Moreira, "Fully polarimetric highresolution 3-D imaging with circular SAR at L-band," IEEE Trans. Geosci. Remote Sens., vol. 52, no. 6. pp. 3074-3090, June 2014.

[2] T. Watanabe, H. Yamada, and Y. Oishi, "First demonstration of spectral domain polarimetric decomposition with airborne circular synthetic aperture radar," IEICE Commun. Express, vol. 9, no. 10, pp. 476-481, 2020.

[3] N. Hayashi and M. Sato, " $F-k$ filter designs to suppress direct waves for bistatic ground penetrating radar," IEEE Trans. Geosci. Remote Sens., vol. 48, no. 3, pp. 1433-1444, Mar. 2010.
[4] J. R. Gallion and R. Zoughi, "Millimeter-Wave Imaging of SurfaceBreaking Cracks in Steel With Severe Surface Corrosion," IEEE Trans. Instrum. Meas., vol. 66, no. 10, pp. 2789-2791, Oct. 2017.

[5] M. Dvorsky, M. T. A. Qaseer, and R. Zoughi, "Detection and Orientation Estimation of Short Cracks Using Circularly Polarized Microwave SAR Imaging," IEEE Trans. Instrum. Meas., vol. 69, no. 9, pp. 7252-7263, Sep. 2020.

[6] T. Watanabe and H. Yamada, "Orientation Estimation of Surface Cracks in Metals Based on Intensity Maximization of Polarimetric Circular Synthetic Aperture Radar Images," IEEE Trans. Instrum. Meas., to be published.

[7] T. Vaupel and T .F. Eibert, "Comparison and application of near-field ISAR imaging techniques for far-field radar cross section determination," IEEE Trans. Antennas Propag., vol. 54, no. 1, pp. 144-151, Jan. 2006.

[8] A. Osipov, H. Kobayashi, and H. Suzuki, "An Improved Image-Based Circular Near-Field-to-Far-Field Transformation," IEEE Trans. Antennas Propag., vol. 61, no. 2, pp. 989-993, Feb. 2013.

[9] T. Watanabe and H. Yamada, "Far-Field Radar Cross-Section Determination From Near-Field 3-D Synthetic Aperture Imaging With Arbitrary Antenna Scanning Surfaces," 2021, [Online]. Available: 10.36227/techrxiv.16802419.

[10] C. Ozdemir, R. Bhalla, L. C. Trintinalia, and H. Ling, "ASAR-antenna synthetic aperture radar imaging," IEEE Trans. Antennas Propag., vol. 46, no. 12, pp. 1845-1852, Dec. 1998.

[11] C. Ozdemir and H. Ling, "ACSAR-Antenna Coupling Synthetic Aperture Radar Imaging Algorithm," J. Electromagn. Waves Appl., vol. 13, no. 3, pp. 285-306, Jan. 1999.

[12] T. Watanabe and H. Yamada, "Synthetic Aperture Imaging of NearField Scatterers Mutually Coupled With an Antenna Array," IEEE Trans. Instrum. Meas., vol. 70, pp. 1-18, 2021.

[13] T. Watanabe and H. Yamada, "Microwave 3D imaging of near-field scatterers mutually coupled with an antenna array," IEICE Commun. Express, to be published. 